
\title{
25 Research Square \\ Buffalo Milk Can Rescue Fatigue in Young Mice, Alleviate Oxidative Stress and Boost Learning and Memory in Aging Mice
}

\section{Xinglv Liao}

Huazhong Agriculture University

Muhammad Jamil Ahmad

Huazhong Agriculture University

Chao Chen

Huazhong Agriculture University

Jitu W George

University of Nebraska Medical Center

Liguo Yang

Huazhong Agriculture University

Guohua Hua ( $\nabla$ huaguohua09@gmail.com )

Huazhong Agriculture University https://orcid.org/0000-0002-6900-1023

Research

Keywords: buffalo milk, antioxidant, anti-fatigue, learning and memory, aging mice

Posted Date: August 3rd, 2020

DOl: https://doi.org/10.21203/rs.3.rs-48866/v1

License: (1) This work is licensed under a Creative Commons Attribution 4.0 International License.

Read Full License 


\section{Abstract}

Background: An advanced age associated pathology is decreasing cognitive ability and impeding antioxidant system integrity. Adverse drug reactions have prompted the need for complementary and alternative medicine dietary therapy. Buffalo milk is reported to have high levels of various nutrients which makes it an ideal candidate for complementary dietary therapy. However, its effects on oxidation, fatigue, learning and memory potential remains to be explored.

Results: Compared with cow milk, buffalo milk showed better anti-fatigue effect evidenced by improved force swimming time, elevated lactate dehydrogenase, hepatic glycogen, and decline of blood urea nitrogen $(P<0.05)$, especially in young female mice. Pasteurized buffalo milk (PBM) and high-temperature treatment sterilized buffalo milk (HTBM) both rescued the serum and liver anti-oxidative enzymes impaired by D-galactose treatment. However, PBM supplement showed higher liver superoxide dismutase $(P<0.05)$ and glutathione peroxidase level $(P<0.05)$ than HTBM in $D$-galactose induced aging mice. PBM and HTBM both enhanced the priority and discrimination indexes for object recognition and locations tests in D-galactose induced aging mice model. Supplement of PBM or HTBM successfully restored brain superoxide dismutase, glutathione peroxidase levels, and brain protein content in D-galactose induced aging mice $(P<0.05)$. Meanwhile, the elevated malondialdehyde levels were also decreased $(P<0.05)$ by either PBM or HTBM treatment.

Conclusion: Our present study established that buffalo milk, as a natural dairy product, could alleviate fatigue in young mice, and rescue oxidative stress and promote learning and memory in aging mice. Pasteurized buffalo milk showed more favorable effects on aging mice when compared with hightemperature treatment sterilized buffalo milk.

\section{Background}

Aging, either pathological or physiological, is an intricate biological process, leading to the debilitation of tissues and organs culminating in multiple chronic diseases and contributing to morbidity and mortality [1]. One of the accepted theories for multifaceted and complicated aging process is oxidative stress [2, 3]. Reactive oxygen species (ROS) such as hydroxyl radicals, superoxide anions, and hydrogen peroxide $\left(\mathrm{H}_{2} \mathrm{O}_{2}\right)$ are natural byproducts of cellular metabolism [4,5]. Enhanced levels of these ROS by-products beyond antioxidant capacity damage cell membrane, alters cellular permeability and induces cytotoxic reaction. Accordingly, aging increases susceptibility to diseases including vascular aging disorders [6, 7], diabetes [8], muscle dysfunction [9], macular degeneration [10], Alzheimer's disease (AD) [11, 12], skin diseases [13], and a series of other diseases [14, 15].

Fueled by increased lifespan and low fertility, it is estimated that individuals 60 and over will account for $32.2 \%$ of the total population by 2100 [16]. A rise in the global aging population is coupled with a high prevalence of aging-associated diseases [17] and conditions, that can cause fatigue, decreased resistance, and a decline in learning and memory function. Hence, aging-related diseases are a serious 
threat to human health and low quality of life among elderly people. Several drugs are available for reversing learning and memory loss induced by aging. However, aging also leads to altered pharmacokinetics in the elderly leading to adverse drug reactions (ADRs) [18], including palatability, and swallowing [19]. Many studies highlighted that, like conventional medications, alternative remedies can have biological effects and suggested oral liquid formulations for older adults to reduce palatability and swallowing [19].

D-galactose (D-gal), a stimulant of natural aging in mice is widely used for anti-aging and tissue damage research $[20,21]$. Within normal threshold D-gal a reducing sugar and natural constituent of the body completely metabolized in the body. Beyond normal threshold, D-gal is converted to aldose hydrogen peroxide and galactose oxidase in the body, leading to the rapid generation of superoxide anion and oxygen-derived free radicals [22]. Studies have reported that D-gal-induced oxidative stress is involved in cognitive impairment, neurotoxicity, tissue injury, and inflammation [23].

Buffalo milk is the second-largest source of milk supply and has high levels of fat, protein, ash, and lactose than cow milk, and is rich in calcium, magnesium, tocopherol (vitamin E), taurine, free amino acids, and conjugated linoleic acid [24]. The high nutritive value of buffalo milk indicates its potential as growth and health promoter. Nevertheless, systemic research concerning buffalo milk function as antioxidant, anti-fatigue, and effect on learning and memory remains to be explored. In this study, we employed a young and aging mice model to test the viability of buffalo milk on anti-fatigue, and antioxidant activity and its effect on learning and memory, respectively.

\section{Materials And Methods}

\section{Chemicals and reagents}

The assay kits for BUN (Cat.: C013), LDH (Cat.: A020), hepatic glycogen (Cat.: A043), SOD (Cat.: A001), GSH-Px (Cat.: A005), and MDA (Cat.: A005) were purchased from Nanjing Jincheng Biotechnology Institute (Nanjing, China). Vitamin C (Vc), Taurine (Tau) and Acetamide Pyrrolidone (AP) were purchased from Sinopharm Chemical Reagent Co. Ltd (Shanghai, China). To induce the aging mice model, Dgalactose (Cat.: G0750) was purchased from Sigma-Aldrich (St Louis, MO, USA). Other than, the aforementioned chemicals used were of analytical grade.

\section{Animals and experimental design}

For this study, ethical approval (Approval ID: SCXK (Hubei) 20080005) was granted by the Hubei Research Center of Experimental Animals. All experimental protocols executed were in accordance with the guidelines of the Committee of Animal Research Institute, Huazhong Agricultural University, China. Kunming strain (KM) mice obtained from the Hubei Provincial Center for Disease Control were bred at the experimental animal center of Huazhong Agricultural University provided $12 \mathrm{~h}$ light/dark cycle with water and food ad libitum. 
In the anti-fatigue experiment, after one week of adoption time, 4-weeks old males $(n=80)$ and females $(n=80)$ mice were placed into four groups, including control, taurine (Tau), pasteurized buffalo milk (PBM), and pasteurized cow milk groups. Each group included 20 mice. Half of each group $(n=10)$ was used for detecting forced swimming time and changes in physiological and biochemical indexes, respectively. The control and Tau group mice received free drinking water and the taurine aqueous solution respectively, while PBM and PCM group were given free drinking pasteurized buffalo or cow milk for four weeks, respectively. Then forced swimming time was determined using exhaustive swimming exercise (ESE) test and biochemical index was performed.

During an antioxidant and learning aptitude and memory experiments, after one week of adoption time, a total $(n=100) 4$-week-old mice were divided into 10 groups $(n=10)$, five groups for each experiment. The four groups (control, D-gal, D-PBM, and D-HTBM) were the same between the two experiments except for positive control D-Vc and D-AP for antioxidants, and learning and memory, respectively. Except for control (physiological saline solution: $0.1 \mathrm{ml} / 10 \mathrm{gm} /$ day) other groups received D-gal (800 mg/kg/day) subcutaneously in the back of the neck up for 8 weeks up to week 12. During the 8-weeks experiment the control, D-gal, D-Vc, D-AP, D-PBM, and D-HTBM group were given free drinking water, PBM (11.50 \pm $0.04 \mathrm{ml} /$ day), HTBM $(11.59 \pm 0.03 \mathrm{ml} /$ day $)$, aqueous solution of $\mathrm{Vc}(200 \mathrm{mg} / \mathrm{kg} /$ day $)$ and acetamidepyrrolidone ( $400 \mathrm{mg} / \mathrm{kg} /$ day), respectively. The biochemical index and the behavioral test (ORT \& OLT) were performed when the mice were 12 weeks old.

\section{D-galactose induced aging mouse model}

We induced aging mouse model using D-gal ( $800 \mathrm{mg} / \mathrm{kg} /$ day) subcutaneously behind the neck for four weeks consecutively. Biochemical index (SOD \& GSH-Px) for serum, liver, and brain and MDA contents were detected to confirm that aging mouse model was constructed successfully.

\section{Exhaustive swimming exercise (ESE) test and forced swimming time}

To determine force swimming time, after four weeks of treatment, with slight modification, the ESE test was used to evaluate the exercise performance [25]. Mouse from each group was taken out after 12 hours fasting, for swimming exercise, before loaded ( $5 \%$ of individual body weight) with the constant pressure (lead fish sinkers, attached to the tail). The mice were individually carried out in a square swimming pool $(60 \times 45 \times 45 \mathrm{~cm})$ with $30-\mathrm{cm}$ water depth maintained at $25 \pm 1{ }^{\circ} \mathrm{C}$. For measuring the strength of each mouse, swimming time was recorded from the beginning to exhaustion characterized by loss of coordinated movements and failure to return to the surface within 7 seconds. The swimming period was defined as the time spent for floating, struggling, and making basic movements until exhaustion and possible drowning.

\section{Biochemical index determination}

For biochemical analysis of the brain, liver, and serum, mice were sacrificed by cervical dislocation to collect liver and brain tissue. Cerebral cortex and liver were dissected; tissues were rinsed with the ice-cold 
isotonic solution and homogenized in ice-cold physiological saline. Homogenates (10\%) were centrifuged at $4000 \times \mathrm{g}$ at $4{ }^{\circ} \mathrm{C}$ for $10 \mathrm{~min}$, and supernatants were used for biochemical analysis. Blood samples were collected through orbital enucleation. Fresh blood sample was allowed to stand at room temperature for 2 hours, followed by centrifuging at $4000 \times \mathrm{g}$ at $4{ }^{\circ} \mathrm{C}$ for $10 \mathrm{~min}$, and the supernatants were used for biochemical analysis. SOD, GSH-Px, LDH, BUN, MDA, and hepatic glycogen were detected using the kit method following the manufacturer's instructions.

\section{Behavioral test}

Object recognition test (ORT) and object location test (OLT) were performed by slightly modifying the method described by Murakami Y et al. [26]. The apparatus used was of poly (vinyl chloride) made square arena $(40 \times 28 \times 20 \mathrm{~cm})$, walls and floor were painted gray and white, respectively. The recognition objects were of different shapes and visually distinguishable. For avoiding the build-up of olfactory cues, $75 \%$ ethanol was used to clean the boxing arena with objects. The animals were exposed to the empty box in three sessions of 10-min each, to adapt the apparatus and test room. The two phases of ORT, a sample phase trial and a test phase trial had a $1 \mathrm{~h}$ interval. In the former phase trial, following first $5 \mathrm{~min}$ in box, each mouse was exposed to two identical sample stimuli, objects $A$ and $B$ (each $3 \times 3 \times 6 \mathrm{~cm}$ white cubes) for the next $5 \mathrm{~min}$, and the total time spent to explore the two objects and returned to its cage was recorded. In the later phase trial, one of the two objects is replaced by object C (the cylinder is $3 \mathrm{~cm}$ in diameter and $6 \mathrm{~cm}$ high) (Figure S1), and the time difference for exploring each of the objects was recorded.

The sample phase trials of the OLT were similar to those of the ORT, with a slightly different in the size and shape of objects (two cylinders: A and B, $3 \mathrm{~cm}$ diameter and $5 \mathrm{~cm}$ height each). In the test phase trial, their identical copies, one in the same position, replaced the objects, whereas the other one was moved to the adjacent corner to change the position diagonally. In the test phase trials, both objects were equally familiar with the animals, but the location was changed for one (Figure S2). The mice were exposed to the objects for $5 \mathrm{~min}$ and the total time spent exploring each of the two objects was measured.

A discrimination index (DI) and priority index (PID) was calculated according to the following equation:

$D I=(T n-T f) /(T f+T n)$

$P I D=T n /(T f+T n)$

Where Tn and Tf stand for the time spent within 5 mint to explore new and familiar objects, respectively.

\section{Statistical analysis}

All the experimental data were presented as mean \pm S.EM. The Student t-test and One-way analysis of variance (ANOVA) were used to determine the statistically significant difference between, and among the experimental groups using SPSS software version 20 (SPSS Inc., Chicago, IL, USA). Different letters illustrate the significant differences (LSD test) between samples $(P<0.05)$. 


\section{Results}

\section{Buffalo milk is more effect to rescue fatigue than cow milk in young adult mouse model}

Many cross-sectional studies infer that females have a higher capacity to resist fatigue than males [27]. Herein, we studied the anti-fatigue effects of buffalo milk in female and male mice, separately. In male mice, anti-fatigues effects of pasteurized buffalo milk (PBM) were compared with pasteurized cow milk (PCM) (Fig. 1). Taurine (Tau) can increase the exercise tolerance, and reduce exercise-induced fatigue by modulating pro-inflammatory factors [28], so we employed Taurine as a positive control. Bodyweight curve identified that PBM treated mice had a trend of the slight increase in body weight than control, PCM, and Tau groups (Fig. 1a). Forced swimming time and biomarkers such as hepatic glycogen, blood urea nitrogen (BUN), and lactate dehydrogenase (LDH) concentration were used as indicators to evaluate anti-fatigue capacity $[29,30]$. There was no significant difference in forced swimming time among the four groups of male mice $(P>0.05)$ (Fig. 1b). In male mice, LDL levels in PCM group is lower than Tau and PBM groups (Fig. 1C). BUN concentrations of PBM and Tau groups was significantly lower than that of control and PCM groups $(P<0.05)$ (Fig. 1d). Hepatic glycogen content of PBM groups were significantly increased in the Tau group when compared with the PCM group $(P<0.05)($ Fig. 1e).

In female mice, the average bodyweight of the PBM group was slightly higher, but there were no significant differences with other groups $(P>0.05)$ (Fig. 2a). Compared with the control group, the forced swimming time in the Tau and PBM groups increased significantly $(P<0.05)$ (Fig. 2b). The serum LDH concentration of Tau, PBM, and PCM groups was elevated compared to the control group $(P<0.05)$ (Fig. 2C). The BUN concentration was significantly lower in all treatment groups than control and the PBM group exhibiting lowest BUN levels (Fig. 2d). Hepatic glycogen content among the four groups was nonsignificant $(P>0.05)$ (Fig. 2e). These results indicate that pasteurized buffalo milk had a better antifatigue effect on female mice.

\section{Pasteurized buffalo milk has better antioxidant effects than high temperature sterilized buffalo milk in D- galactose induced aging mice model}

Aging reduces the efficiency of anti-oxidative enzymes rendering older people more susceptible to free radical stress and oxidative insult [31]. Chronic exposure of Dgalactose (D-gal) is a well-established model to induce accelerated aging in mice [32]. Based on better antifatigue effects of PBM in in young mice model, we employed D-gal induced aging model to evaluate whether PBM or/and high temperature sterilized buffalo milk (PTBM) could rescue oxidative stress induced by aging. Antioxidant enzymes such as superoxide dismutase (SOD), glutathione peroxidase (GSH-Px) function as scavengers for superoxide and hydrogen to inhibit ROS-induced damage and serve as biomarkers of oxidative stress [33]. D-gal treatment significantly decreased SOD $(P<0.05)$ and GSH-Px $(P<0.05)$ levels in serum, whereas levels of MDA were increased $(P<0.05)$. Besides, the GSH-Px was also significantly downregulated, and MDA was upregulated in liver tissue (Fig. 3). These indicated the successful establishment of the D-gal induced aging mouse model. Vitamin $\mathrm{C}(\mathrm{Vc})$ which has potent antioxidant and scavenging ability of hydroxyl radical and superoxide anion [34], was used as a positive control. There was no difference between the 
average daily consumption between pasteurized and high temperature sterilized buffalo milk (Table S1). Nonetheless, the total body weight gains of D-PBM increased significantly than the D-gal group $(P<0.05)$ (Table 1), while other groups had no significant difference. These results indicated that PBM has more favorable bodyweight competence in D-gal induced aging mice.

Table 1

Effect of pasteurized and high temperature sterilized buffalo milk on body weight of ageing mice

\begin{tabular}{|c|c|c|c|c|c|}
\hline $\begin{array}{l}\text { Groups } \\
(n=10)\end{array}$ & $\begin{array}{l}\text { Initial } \\
\text { weight } \\
\text { (g) }\end{array}$ & $\begin{array}{l}0-4 \text { weeks } \\
\text { weight gain (g) }\end{array}$ & $\begin{array}{l}4-8 \text { weeks } \\
\text { weight gain (g) }\end{array}$ & $\begin{array}{l}\text { Total weight } \\
\text { gain }(\mathrm{g})\end{array}$ & $\begin{array}{l}\text { Weight gain compared } \\
\text { to control (\%) }\end{array}$ \\
\hline Control & $\begin{array}{l}24.45 \pm \\
0.57^{\mathrm{a}}\end{array}$ & $6.93 \pm 0.61^{\mathrm{ab}}$ & $2.93 \pm 0.71^{a b}$ & $\begin{array}{l}9.86 \pm \\
1.19^{\mathrm{ab}}\end{array}$ & -- \\
\hline D-gal & $\begin{array}{l}24.47 \pm \\
0.51^{\mathrm{a}}\end{array}$ & $6.89 \pm 0.45^{\mathrm{ab}}$ & $1.57 \pm 0.16^{a}$ & $8.46 \pm 0.45^{\mathrm{a}}$ & $-14.20 \%$ \\
\hline $\mathrm{D}-\mathrm{Vc}$ & $\begin{array}{l}24.48 \pm \\
0.60^{\mathrm{a}}\end{array}$ & $6.45 \pm 0.53^{a}$ & $2.05 \pm 0.21^{\mathrm{ab}}$ & $8.5 \pm 0.57^{a}$ & $-13.79 \%$ \\
\hline D-PBM & $\begin{array}{l}24.56 \pm \\
0.56^{a}\end{array}$ & $8.44 \pm 0.74^{b}$ & $3.04 \pm 0.73^{b}$ & $\begin{array}{l}11.47 \pm \\
1.38^{\mathrm{b}}\end{array}$ & $16.36 \%$ \\
\hline D-HTBM & $\begin{array}{l}24.38 \pm \\
0.49^{a}\end{array}$ & $7.54 \pm 0.72^{\mathrm{ab}}$ & $2.75 \pm 0.35^{\mathrm{ab}}$ & $\begin{array}{l}10.28 \pm \\
0.72^{\mathrm{ab}}\end{array}$ & $4.28 \%$ \\
\hline
\end{tabular}

We next assessed serum antioxidant effects of buffalo milk on biochemical parameters related to antioxidant competence in aging mice (Fig. 3). Compared with the D-gal group, the serum SOD activity of the D-Vc group, D-PBM group, and the D-HTBM group increased significantly $(P<0.05)$ in the serum (Fig. 3a). Vc, PBM, and HTBM treatments all diminished elevated MDA levels induced by D-gal (Fig. 3c). The serum MDA level of the D-PBM group was significantly lower than the D-Vc group (Fig. 3c). Compared with the D-gal group, the activity of GSH-Px in the serum of the D-gal induced aging mice supplied with Vc (D-Vc), PBM (D-PBM), or HTBM (D-HTBM) groups significantly increased $(P<0.05)$ (Fig. 3e). PBM and Vc treatment restored the GSH-Px to control levels (Fig. 3e). However, GSH-Px in the HTBM group was significantly lower than the control group $(P<0.05)$ (Fig. 3e).

We then detected the effects of buffalo milk related to oxidative stress in the liver in our aging mouse model. The liver SOD activity of the D-PBM group significantly increased when compared with all the other groups $(P<0.05)$ (Fig. 3b). Both Vc, PBM, and HTBM were able to decrease MDA concentrations to basal levels (Fig. 3d), indicating that $V_{c}$ and buffalo milk can alleviate the age-induced increase of MDA. Compared with the D-gal group, liver GSH-Px activities of D-Vc, D-PBM groups were significantly increased $(P<0.05)$, but HTBM treatment failed to reinforce D-gal induced decline of GSH-Px (Fig. 3f). 
These results showed that buffalo milk and $V_{c}$ had boosted antioxidant effects on aging mice by increasing SOD, GSH-Px content, and decreasing MDA levels. More importantly, pasteurized buffalo milk has a better antioxidant effect than high-temperature buffalo milk.

\section{Pasteurized and high-temperature sterilized buffalo milk boosted learning and memory in D-galactose induced aging mice}

We next evaluated the effect of buffalo milk on learning and memory capacity by employing the Dgalactose induced aging mice model. The physiological basis of animal learning and memory ability is a conditioned reflex. The main conditioned reflex tests include passive avoidance task, spontaneous novel object recognition, and object location recognition, and Morris water-maze procedure [35]. Results of object recognition test (ORT) (Fig. 4a, b), and object location test (OLT) (Fig. 4c, d) showed that the priority index (PID) and the discrimination index (DI) of the D-gal group were significantly lower than the control group $(P<0.05)$. Besides, the D-gal group had significantly lower GSH-PX, SOD, and higher MDA levels than the control group $(P<0.05)$ (Fig. 5$)$, which confirmed the success of the aging mice model.

In the ORT experiment, the PID was significantly increased in D-gal induced aging mice supplied with AP (D-AP), D-PBM, and D-HTBM group (Fig. 4a). Consistently, the discrimination index of the D-gal group was also successfully rescued by AP, PBM, or HTBM treatment (Fig. 4b). In the OLT experiment, compared with the D-gal group, both the DI and PID of the D-AP, the D-PBM, and the D-HTBM groups increased significantly $(P<0.05)$ (Fig. 4c, d). These data suggested that buffalo milk could improve the decline in learning and memory capacity caused by $\mathrm{D}$-gal induced aging.

Subsequently, the biochemical indicators (SOD, GSH-Px, and MDA) related to oxidation in the brain were examined. Compared with the D-gal group, the brain SOD and GSH-Px activities of the D-AP, D-PBM, and D-HTBM groups increased significantly (Fig. 5a, C), while MDA activity was significantly decreased in DAP and D-PBM groups $(P<0.05)$ (Fig. 5b). Finally, we detected the brain protein content in different groups, the brain protein content of the D-gal group was significantly lower than that of the control group while a substantial increase was observed in D-AP (13.38\%), D-PBM (16.20\%), and D-HTBM (14.43\%) compared to the $D$-gal group $(P<0.05)$ (Fig. 5 d).

\section{Discussion}

With ever-growing population, and increased number of elder people ( $>60$ year), global rise of impaired cognitive behavior and integrity of the antioxidant system warns to find natural remedies as medication is complicated in older adults. Biochemical index (LDL, BUN, Hepatic Glycogen, SOD, GSH-Px and MDA) of body fluids and tissues are indicator of fatigue, oxidative stress induce damage, and neurological functions including cognitive behavior and learning and memory [33, 36-38]. In this study, we employed young mice model to demonstrate the anti-fatigue property of buffalo milk, the buffalo milk showed a better fatigue relief effect especially in female young mice. By employing D-galactose induced aging mice 
model, our results supported that, buffalo milk, especially pasteurized buffalo milk, can rescue oxidative stress, and boost learning and memory capacity as evidenced by increased endogenous antioxidants (SOD \& GSH-Px) in serum, liver, and decreased MDA contents.

Many cross-sectional studies have demonstrated that females have a greater capacity to resist fatigue than males [27]. Forced swimming time and biomarkers such as hepatic glycogen, BUN, and LDH concentration are often used as indicators to evaluate anti-fatigue $[29,30]$. In the present study, we found that PBM rescued fatigue more significantly in female than male mice. We observed a decline of BUN in both male and female mice. However, elevated serum LDH and hepatic glycogen were seen only in females that had increased swimming time. It concludes and confirms the effect of sex influence on the anti-fatigue response. LDH activity reflects the rate of lactic acid metabolism, significantly affects the elimination of fatigue, hence high LDH levels indicate that animals are more adaptive to exercise [39]. In the process of strenuous exercise, in addition to glucose, hepatic glycogen is the primary source of energy. Increased liver glycogen reserves are conducive to greater exercise endurance and capacity [40]. This is in line with our results of increased hepatic glycogen contents in the PBM treated group which showed less fatigue. Constant and high-intensity labor results in the accumulation of metabolites in the body and is the main cause of physical fatigue [41]. In our findings, forced swimming time significantly increased with PBM in female mice indicating they were less fatigued. Various studies have identified a broad range of bioactive peptides that have been reported to influence antioxidant activities. Bovine $\beta$ casein (dairy products) derived PGPIPN, an immunomodulatory peptide (Pro-Gly-Pro-Ile-Pro-Asn), can enhance resistance to fatigue [42]. Buffalo milk is rich in casein [24], which may produce more PGPIPN and relieve anti-fatigue effects. In addition, EAMAPK ( $\beta-C N, f 115-120)$, an antioxidant peptide has been identified in buffalo milk and reported to inhibit ROS release and increased antioxidant response [43]. Buffalo dairy products derived peptides VLPVPQK ( $\beta-C N, f 185-191)$ and AVPYPQR ( $\beta-C N, f 192-197)$ have also been identified that possess a strong antioxidant potential [44].

Oxidative stress is associated with multiple disease states and considered to be a primary cause of aging [45]. Excessive ROS can activate endogenous antioxidant defense mechanisms, leading to an increase in antioxidant enzymes especially SOD and GSH-Px. SOD effectively converts 02- into hydrogen peroxide and hydroxyl radical [46]. It balance the ROS and reactive nitrogen levels to limit their potential toxicity and influence physiological changes associated with aging [47]. Free radical-induced tissue damage generates lipid peroxidation by-products such as malondialdehyde (MDA) and modification of proteins with MDA [48], can reflect anti-oxidant levels of the body to some extent.

Our findings conclude that PBM can rescue aging-induced oxidative stress by increasing liver SOD, GSHPx, and declining MDA contents. Moreover, ORT and OLT experiments showed D-PBM could improve decline in age-associated learning and memory capacity. Studies have shown that buffalo milk peptide can improve antioxidant capacity in D-gal model aging mice [49]. Bioactive peptides in fermented buffalo yogurt display increased antioxidant activity [50]. In addition, some $\beta$-casein neuropeptides (LHLPLPL, $\beta$ $\mathrm{CN} \mathrm{f148-154)} \mathrm{in} \mathrm{gastrointestinal} \mathrm{digest} \mathrm{of} \mathrm{dairy} \mathrm{function} \mathrm{as} \mathrm{prolyl} \mathrm{oligo} \mathrm{peptidase} \mathrm{(POP)} \mathrm{inhibitors.} \mathrm{POP}$ inhibitors reverse the depletion of neuropeptide induced by aging or neurodegenerative disorders and 
improve the cognitive and neurological functions. It confirms the possible role of POP in pathological conditions such as Parkinson's and Alzheimer's diseases [51].

\section{Conclusion}

Our study highlight that pasteurized buffalo milk could strengthen the anti-fatigue ability in female young mice, rescue oxidative stress damage, and boost learning and memory in aging mice. Hence, further research is warranted to explore bioactive compounds of buffalo milk with anti-fatigue and boosting effects on learning and memory in humans, as well as underlying mechanisms.

\section{Additional Files}

- Additional File 1: Figure S1. Object recognition test (ORT) design (png.196 KB)

- Additional File 2: Figure S2. Object location test (OLT) design (png.199 KB)

- Additional File 3: Table S1. The mice daily milk consumption in pasteurized buffalo milk group and high-temperature sterilized buffalo milk group (docx $12.6 \mathrm{~KB}$ )

\section{Declarations}

\section{Abbreviations}

PBM

Pasteurized buffalo milk; PCM:Pasteurized cow; HTBM:high-temperature treatment sterilized buffalo milk; ROS):Reactive oxygen species; $\mathrm{H} 2 \mathrm{O2}$ :hydrogen peroxide; AD:Alzheimer's disease; ADRs:adverse drug reactions; D-gal:D-galactose; Tau:Taurine; AP:Acetamidepyrrolidone; Vc:Vitamin C; GSH-Px:Glutathione peroxidase; SOD:Superoxide dismutase; MDA:malondialdehyde; BUN:blood urea nitrogen; LDH:lactate dehydrogenase; ESE:Exhaustive swimming exercise; ORT:Object recognition test; OLT:object location test; DI:Discrimination index; PI:Priority index; POP:prolyl oligo peptidase

\section{Declarations}

\section{Ethics approval and consent to participate}

Ethical approval (Approval ID: SCXK (Hubei) 20080005) was approved by the Hubei Research Center of Experimental Animals. All experimental protocols executed were in accordance with the guidelines of the Committee of Animal Research Institute, Huazhong Agricultural University, China.

\section{Availability of data and material}

The data during and /or analyzed during the current study are available from the corresponding author on reasonable request. 


\section{Competing interests}

The authors declare that they have no competing interests.

\section{Funding}

This work was financial supported by National Science Foundation of China (No. 31872352), Fundamental Research Funds for the Central Universities (2662018PY037, 2662018PY091), Earmarked Fund for Modern Agro-industry Technology Research System (CARS-36), and the Special Major Projects for Technological Innovation in Hubei Province (2017ABA057).

\section{Author Contributions}

Conceptualization \& methodology, X.L and MJA.; software, X.L and C.C.; validation, X.L, C.C. and MJA.; formal analysis, X.L.; investigation, X.L and MJA.; resources, C.C; data curation, MJA and C.C.; writingoriginal draft preparation, C.C and MJA.; writing-review and editing, J.W.G; visualization, X.L and C.C; supervision L.Y and G.H.; project administration, L.Y and GH.; funding acquisition, $L Y$ and GH.

\section{Acknowledgements}

Not applicable.

\section{Author details}

${ }^{1}$ Key Lab of Agricultural Animal Genetics, Breeding and Reproduction of Ministry of Education, College of Animal Science and Technology, Huazhong Agricultural University, Wuhan 430070, China;

2 Olson Centre for Women's Health, Department of Obstetrics and Gynecology, University of Nebraska Medical Centre, Omaha, Nebraska 68198, USA;

3. International Joint Research Centre for Animal Genetics, Breeding and Reproduction, Wuhan, 430070, China;

4. Hubei Province's Engineering Research Centre in Buffalo Breeding \& Products, Wuhan, Hubei, China

\section{References}

1. Bao Q, Pan J, Qi H, Wang L, Qian H, Jiang F, Shao Z, Xu F, Tao Z, Ma Q. Aging and age-related diseases-from endocrine therapy to target therapy. MOL CELL ENDOCRINOL. 2014;394(1-2):115-8.

2. Da Cunha MDSB, Arruda SF. Tucum-do-Cerrado (Bactris setosa Mart.) may promote anti-aging effect by upregulating SIRT1-Nrf2 pathway and attenuating oxidative stress and inflammation. Nutrients. 2017;9(11):1243.

3. Wadhwa R, Gupta R, Maurya PK. Oxidative stress and accelerated aging in neurodegenerative and neuropsychiatric disorder. CURR PHARM DESIGN. 2018;24(40):4711-25. 
4. Liochev SI. Reactive oxygen species and the free radical theory of aging. FREE RADICAL BIO MED. 2013;60:1-4.

5. Sena LA, Chandel NS. Physiological roles of mitochondrial reactive oxygen species. MOL CELL. 2012;48(2):158-67.

6. Barton M, Husmann M, Meyer MR. Accelerated vascular aging as a paradigm for hypertensive vascular disease: prevention and therapy. CAN J CARDIOL. 2016;32(5):680-6. e684.

7. Buford TW. Hypertension and aging. Ageing Res Rev. 2016;26:96-111.

8. Palmer AK, Kirkland JL. Aging and adipose tissue: potential interventions for diabetes and regenerative medicine. EXP GERONTOL. 2016;86:97-105.

9. Domingues-Faria C, Vasson M-P, Goncalves-Mendes N, Boirie Y, Walrand S. Skeletal muscle regeneration and impact of aging and nutrition. AGEING RES REV. 2016;26:22-36.

10. Ma W, Wong WT. (2016). Aging changes in retinal microglia and their relevance to age-related retinal disease. Retinal Degenerative Diseases: Springer), pp. 73-78.

11. Cleeland C, Pipingas A, Scholey A, White D. Neurochemical changes in the aging brain: A systematic review. Neurosci Biobehav Rev. 2019;98:306-19.

12. Maldonado-Lasuncion I, Atienza M, Sanchez-Espinosa MP, Cantero JL. Aging-related changes in cognition and cortical integrity are associated with serum expression of candidate microRNAs for Alzheimer disease. CEREB CORTEX. 2019;29(10):4426-37.

13. Armenta AM, Henkel ED, Ahmed AM. Pigmentation disorders in the elderly. DRUG AGING. 2019;36(3):235-45.

14. Mander BA, Winer JR, Walker MP. Sleep and human aging. Neuron. 2017;94(1):19-36.

15. Vicini C, De Vito A, lannella G, Gobbi R, Corso RM, Montevecchi F, Polimeni A, De Vincentiis M, Meccariello G, D'agostino $G$. The aging effect on upper airways collapse of patients with obstructive sleep apnea syndrome. EUR ARCH OTO-RHINO-L. 2018; 275(12):2983-2990.

16. Lutz W, Sanderson W, Scherbov S. The coming acceleration of global population ageing. Nature. 451(7179):716-719.

17. Liu GH, Ding Z, Belmonte JCl. IPSC technology to study human aging and aging-related disorders. CURR OPIN CELL BIOL. 2012;24(6):765-74.

18. Khalil $\mathrm{H}$, Huang $\mathrm{C}$. Adverse drug reactions in primary care: a scoping review. BMC Health Serv Res. 2020;20(1):5.

19. Belissa E, Vallet T, Laribe-Caget S, Chevallier A, Chedhomme F-X, Abdallah F, Bachalat N, Belbachir SA. Boulaich I and Bloch V. Acceptability of oral liquid pharmaceutical products in older adults: palatability and swallowability issues. BMC GERIATR. 2019;19(1):1-9.

20. Li L, Ng T, Gao W, Li W, Fu M, Niu S, Zhao L, Chen R, Liu F. Antioxidant activity of gallic acid from rose flowers in senescence accelerated mice. LIFE SCI. 2005;77(2):230-40.

21. Chen H-L, Wang C-H, Kuo Y-W, Tsai C-H. Antioxidative and hepatoprotective effects of fructooligosaccharide in D-galactose-treated Balb/cJ mice. BRIT J NUTR. 2011;105(6):805-9. 
22. Cui X, Zuo P, Zhang Q, Li X, Hu Y, Long J, Packer L, Liu J. Chronic systemic D-galactose exposure induces memory loss, neurodegeneration, and oxidative damage in mice: Protective effects of R-alipoic acid. J Neurosci Res. 2006;84(3):647-54.

23. Zhang Z-F, Fan S-H, Zheng Y-L, Lu J, Wu D-M, Shan Q, Hu B. Purple sweet potato color attenuates oxidative stress and inflammatory response induced by $\mathrm{d}$-galactose in mouse liver. FOOD CHEM TOXICOL. 2009;47(2):496-501.

24. Khedkar CD, Kalyankar SD, Deosarkar SS. (2016). Buffalo Milk.

25. Shih-Yi W, Wen-Ching H, Chieh-Chung L, Ming-Fu W, Chin-Shan H, Wen-Pei H, Chia-Chung H, Hsiao-Li C, Chi-Chang H. Pumpkin (Cucurbita moschata) fruit extract improves physical fatigue and exercise performance in mice. Molecules. 2012;17(10):11864-76.

26. Murakami Y, Zhao Q, Harada K, Tohda M, Watanabe H, Matsumoto K. Choto-san, a Kampo formula, improves chronic cerebral hypoperfusion-induced spatial learning deficit via stimulation of muscarinic M 1 receptor. PHARMACOL BIOCHEM BE. 2005;81(3):616-25.

27. Ribeiro AS, Avelar A, Schoenfeld BJ, Trindade MCC, Ritti-Dias RM, Altimari LR, Cyrino ES. Effect of 16 Weeks of Resistance Training on Fatigue Resistance in Men and Women. J HUM KINET. 2014;42(1):165-74.

28. Kato T, Okita S, Wang S, Tsunekawa M, Ma N. (2015). The effects of taurine administration against inflammation in heavily exercised skeletal muscle of rats. Taurine 9: Springer), pp. 773-784.

29. Liu R, Wu L, Du Q, Ren JW, Chen QH, Li D, Mao RX, Liu XR, Li Y. Small Molecule Oligopeptides Isolated from Walnut (Juglans regia L.) and Their Anti-Fatigue Effects in Mice. Molecules. 2019;24(1):15.

30. Li D, Ren JW, Zhang T, Liu R, Wu L, Du Q, Li Y. Anti-fatigue effects of small-molecule oligopeptides isolated from Panax quinquefolium L. in mice. FOOD FUNCT. 2018;9(8):4266-73.

31. Peter V, Werner S. Clinical oxidation parameters of aging. FREE RADICAL RES. 2006;40(12):1339-49.

32. Cui X, Wang L, Zuo P, Han Z, Fang Z, Li W, Liu J. D-galactose-caused life shortening in Drosophila melanogaster and Musca domestica is associated with oxidative stress. BIOGERONTOLOGY. 2004;5(5):317-25.

33. Xu M, Liang R, Li Y, Wang J. Anti-fatigue effects of dietary nucleotides in mice. J FOOD NUTR RESSLOV. 2017;61(1):1334485.

34. Fan W, He J, Ping H, Amp SA. Study on Extraction of Vitamin C from Wild Chenopodium album and Its Antioxidant Activity. J. Food Ind. 2018.

35. Zhao Z, Loane DJ, Murray MG, Stoica BA, Faden Al. Comparing the predictive value of multiple cognitive, affective, and motor tasks after rodent traumatic brain injury. J NEUROTRAUM. 2012;29(15):2475-89.

36. Chi A, Li H, Kang C, Guo H, Wang Y, Guo F, Tang L. Anti-fatigue activity of a novel polysaccharide conjugates from Ziyang green tea. Int J Biol Macromol. 2015;80:566-72.

37. Yang T-C, Chen Y-J, Chang S-F, Chen C-H, Chang P-Y, Lu S-C. Malondialdehyde mediates oxidized LDL-induced coronary toxicity through the Akt-FGF2 pathway via DNA methylation. J BIOMED SCI. 
2014;21(1):11.

38. Wu K-J, Hsieh M-T, Wu C-R, Wood WG, Chen Y-F. Green tea extract ameliorates learning and memory deficits in ischemic rats via its active component polyphenol epigallocatechin-3-gallate by modulation of oxidative stress and neuroinflammation. EVID-BASED COMPL ALT. 2012; 2012.

39. Chao M, Xiao K, Zheng S, Mu S, Fang W, Xu B. Anti-fatigue Activity of MRJPs from Fresh Royal Jelly in Mice. Zhongguo Shipin Xuebao 2017.

40. Laurent D, Yerby B, Zhang B, Chen XH, Gounarides J, Zhang J, Gao J, Bebernitz G, Duttaroy A. Hepatic glycogen cycling contributes to glucose lowering effects of the glucokinase activator LCZ960. Eur J Pharmacol. 2013;715(1-3):89-95.

41. Bao L, Cai X, Wang J, Zhang Y, Sun B, Li Y. Anti-Fatigue Effects of Small Molecule Oligopeptides Isolated from Panax ginseng C. A. Meyer in Mice. NUTRIENTS. 2016;8(12):807.

42. Fang GU, Qin YD, Dong HS, Su-Ping LI, Liu H, Bin Y. EFFECTS OF IMMUNOMODULATING PEPTIDE FROM $\beta$-CASEIN ON ANTI-OXIDATION AND ANTI-FATIGUE IN MICE. Acta Nutrimenta Sinica. 2006.

43. Basilicata MG, Pepe G, Sommella E, Ostacolo C, Manfra M, Sosto G, Pagano G, Novellino E, Campiglia P. Peptidome profiles and bioactivity elucidation of buffalo-milk dairy products after gastrointestinal digestion. Food Res Int. 2018;105:1003-10.

44. Rival SG, Fornaroli S, Boeriu CG, Wichers HJ. Caseins and casein hydrolysates. 1. Lipoxygenase inhibitory properties. J AGR FOOD CHEM. 2001;49(1):287-94.

45. Halliwell B. Oxidants and human disease: some new concepts. FASEB J. 1987;1(5):358-64.

46. Alfonso V, Champy R, Mitrovic D, Collin P, Lomri A. Reactive oxygen species and superoxide dismutase: Role in joint disease. Joint Bone Spine. 2007;74(4):324-9.

47. Wang Y, Branicky R, Noë A, Hekimi S. Superoxide dismutases: Dual roles in controlling ROS damage and regulating ROS signaling. J CELL BIOL. 2018;217(6):1915-28.

48. Joohee K, Chehade J, Pinnas JL, Mooradian AD. Effect of select antioxidants on malondialdehyde modification of proteins. Nutrition. 2000;16(11):1079-81.

49. Ling LI, Tang Y, Nong HR, Zeng QK, Lin B, Ying Z. Antioxidative effect of buffalo milk peptide in subacute aging model mice. INT J FOOD SCI TECH. 2012.

50. Taha S, Abd ME, Gobba CD, Abdel-Hamid M, Khalil E, Hassan D. Antioxidant and antibacterial activities of bioactive peptides in buffalo's yoghurt fermented with different starter cultures. FOOD SCI BIOTECHNOL. 2017;26(5):1-8.

51. Myöhänen TT, García-Horsman JA, Tenorio-Laranga J, Männistö PT. Issues about the physiological functions of prolyl oligopeptidase based on its discordant spatial association with substrates and inconsistencies among mRNA, protein levels, and enzymatic activity. J HISTOCHEM CYTOCHEM. 2009;57(9):831-48.

\section{Figures}



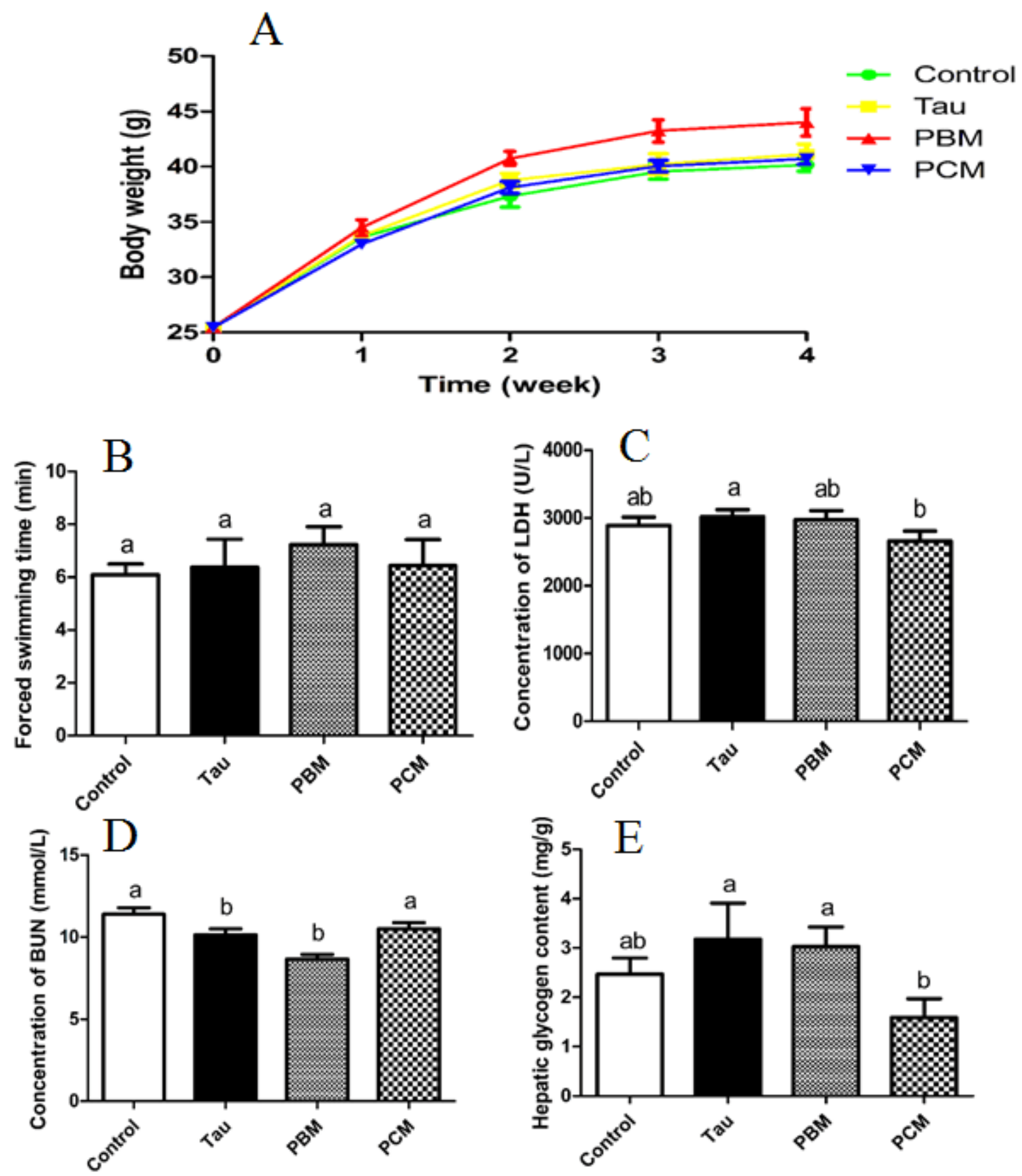

Figure 1

Effect of pasteurized buffalo milk and cow milk on the anti-fatigue index of male mice. a Male ice body weight curve in different treatment groups. b Forced swimming time in different treatment groups. $\mathrm{c}$ Lactate dehydrogenase (LDH), d blood urea nitrogen (BUN) and e hepatic glycogen content in the male mice serum from control, and taurine (Tau), pasteurized buffalo milk (PBM), pasteurized cow milk (PCM) treatment groups. Data are expressed as means \pm S.E.M $(n=20)$. Different letters illustrate the significant differences between groups $(P<0.05)$. 

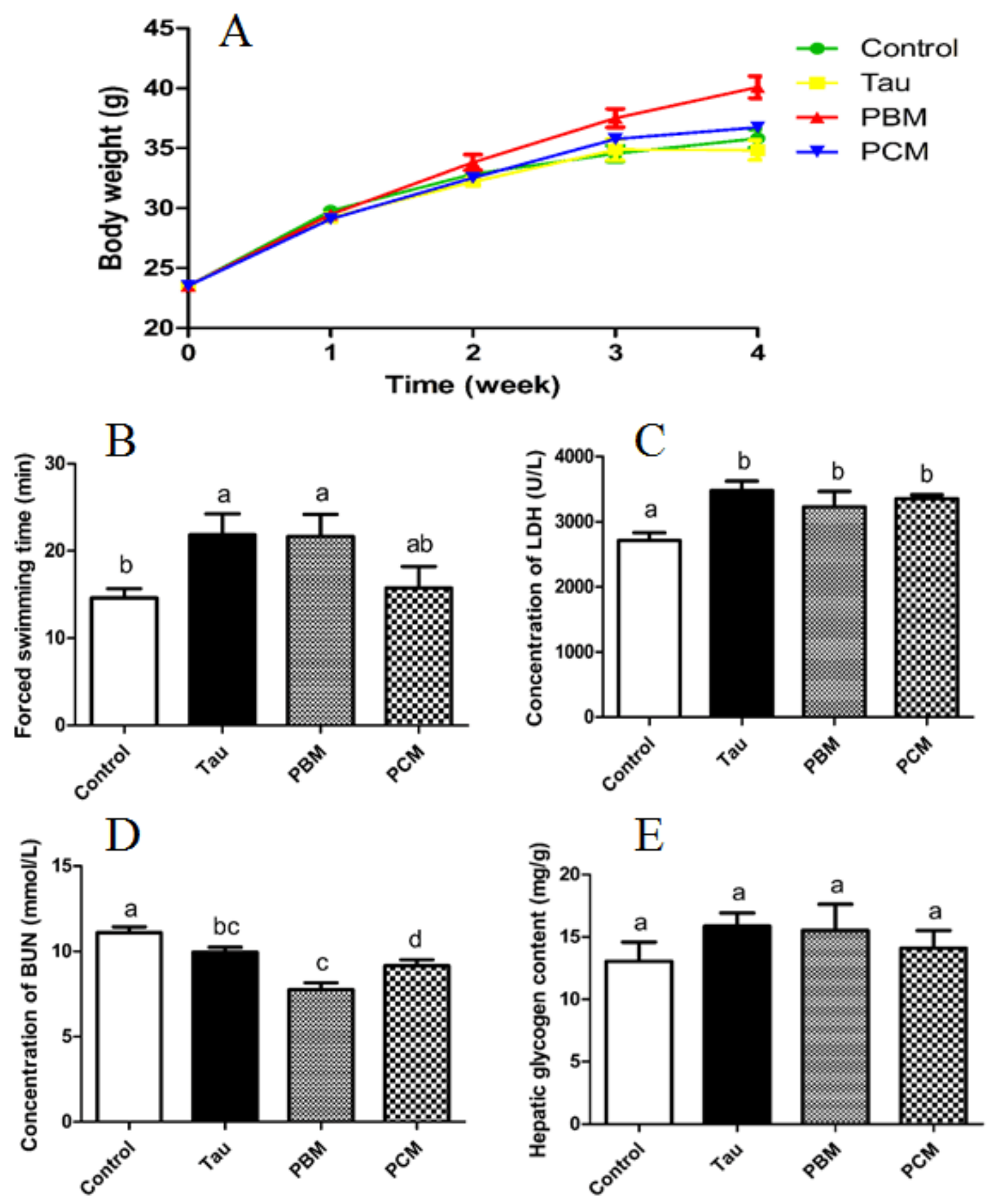

Figure 2

Effect of pasteurized buffalo and cow milk on the anti-fatigue index of female young mice. a Female mice body weight curve in different treatment groups. $b$ Forced swimming time in different treatment groups. c Lactate dehydrogenase (LDH), d blood urea nitrogen (BUN), and e hepatic glycogen content in the female mice serum from control, and taurine (Tau), pasteurized buffalo milk (PBM), pasteurized cow milk (PCM) treatment groups. Data are expressed as means \pm S.E.M $(n=20)$. Different letters illustrate the significant differences between groups $(P<0.05)$. 


\section{Serum}
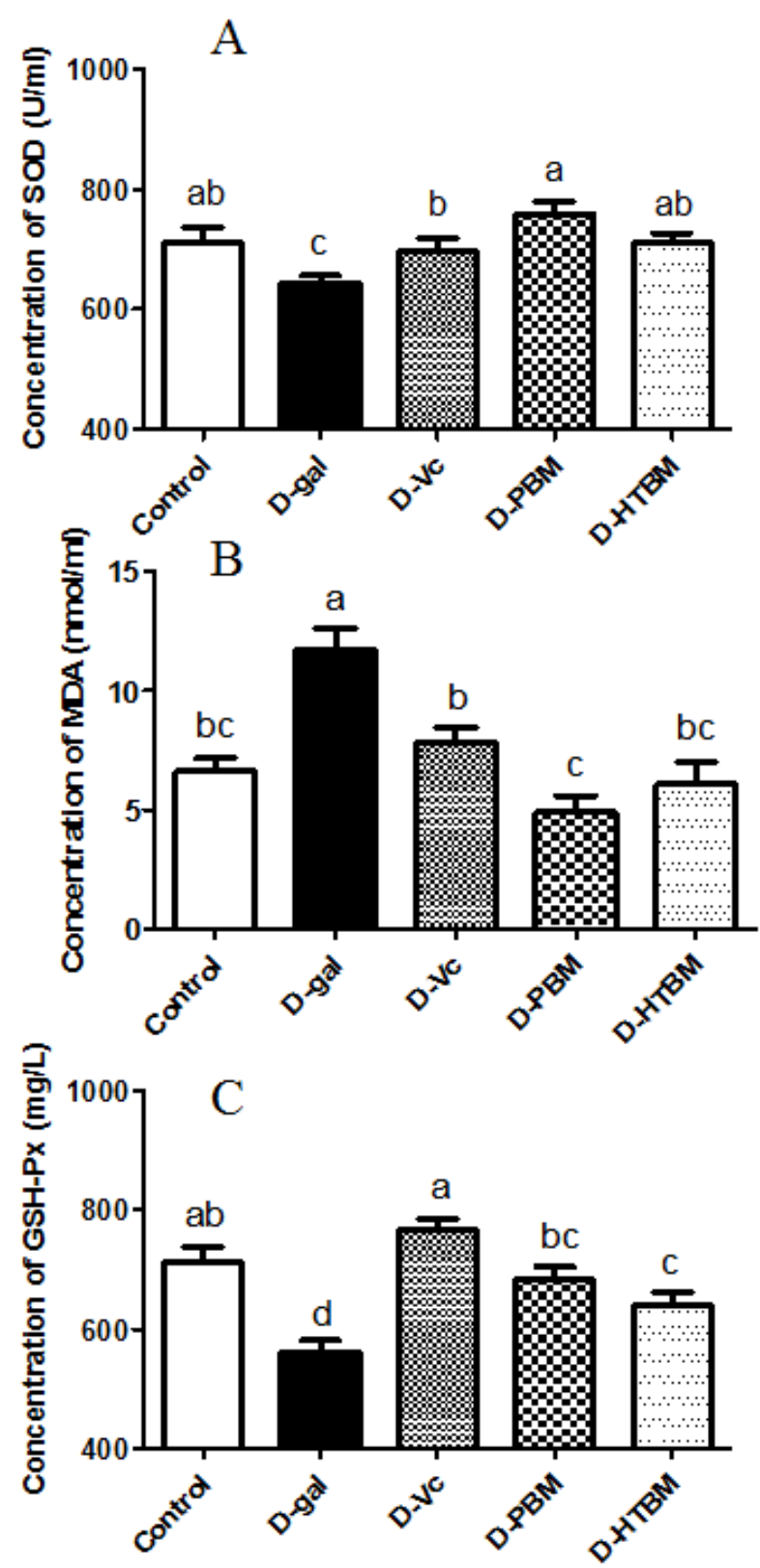

Liver
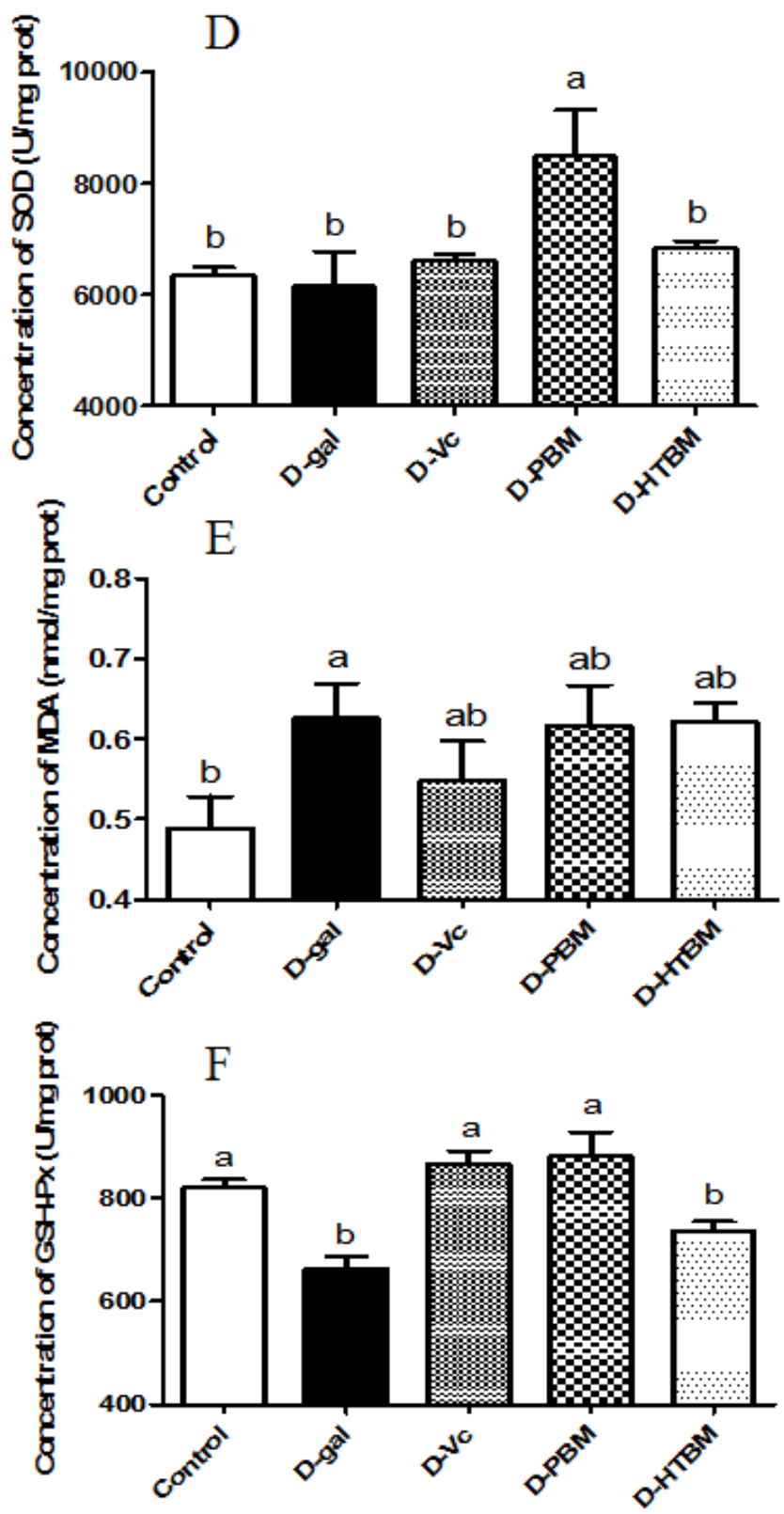

Figure 3

Effects of pasteurized and high temperature sterilized buffalo milk on biochemical parameters related to oxidants in serum and liver in D-galactose (D-gal) induced aging mice. a Serum and b liver superoxide dismutase (SOD) levels in different treatment groups. c Serum and d liver malondialdehyde (MDA) levels in different treatment groups. e Serum and $\mathrm{f}$ liver glutathione peroxidase (GSH-Px) levels in different treatment groups. Data are expressed as means \pm S.E.M $(n=10)$. Different letters illustrate the significant differences between group $(P<0.05)$. 

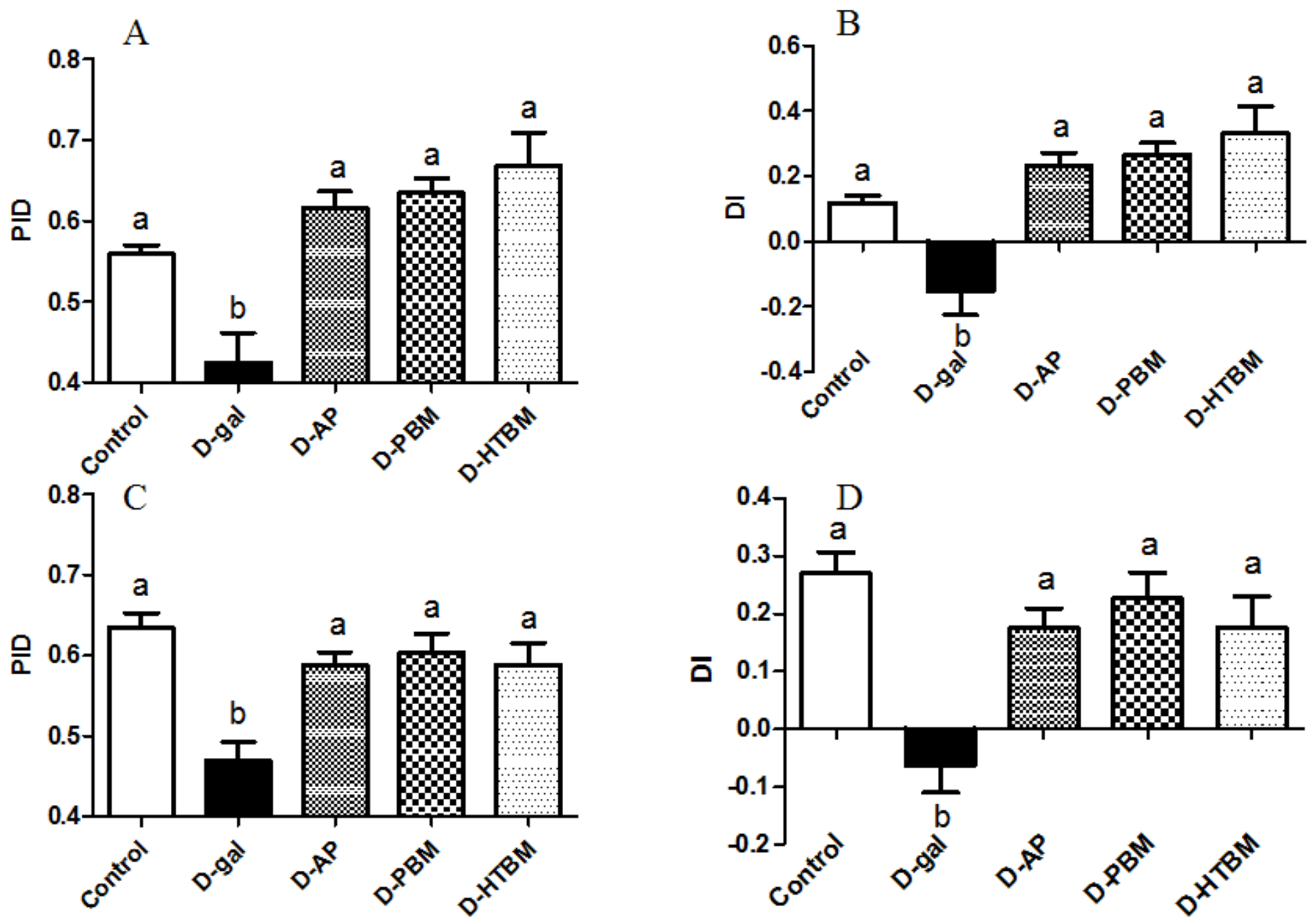

Figure 4

Effects of pasteurized and high-temperature sterilization buffalo milk on object recognition test (ORT) and object location test (OLT) of D-galactose aging mice. a Priority index (PID), b discrimination index (DI) indicated by ORT. c PID, and d DI indicated by OLT. Data are expressed as means \pm S.E.M $(n=10)$. Different letters illustrated the significant differences between samples $(P<0.05)$. 

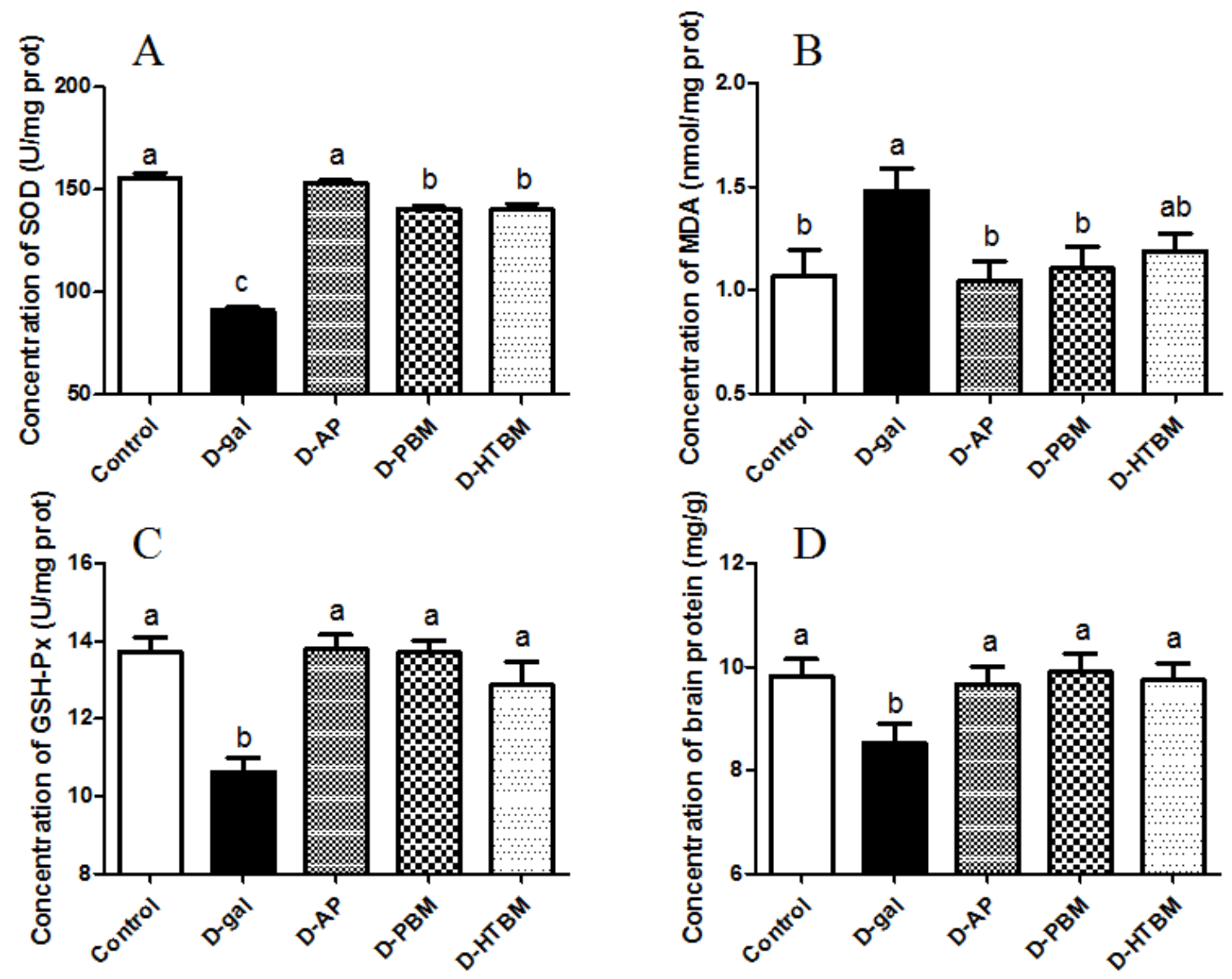

Figure 5

Effects of pasteurized and high temperature sterilized buffalo milk on brain biochemical parameters related to oxidantative stress in D-galactose induced aging mice. a Brain Superoxide dismutase (SOD) level, b Malondialdehyde (MDA) level, c glutathione peroxidase (GSH-Px) level, and d brain protein content in control, D-galactose (D-gal), D-gal induced aging mice supplied with acetamide pyrrolidone (D-gal + $A P)$, pasteurized buffalo milk (D-gal $+P$ ) or high-temperature sterilized buffalo milk (D-gal $+H T)$. Data are expressed as means \pm S.E.M $(n=10)$. Different letters illustrated the significant differences between samples $(P<0.05)$.

\section{Supplementary Files}

This is a list of supplementary files associated with this preprint. Click to download. 
- Supplemental.docx

Page 20/20 